\section{Hierarchical Order in Hollow Spheres of Mesoporous Silicates}

\author{
Hong-Ping Lin, Yah-Ru Cheng, and \\ Chung-Yuan Mou*
}

Department of Chemistry, National Taiwan University, Taipei, Taiwan

Received J uly 14, 1998

Revised Manuscript Received October 5, 1998

The preparation of mesoporous aluminosilicate materials was recently el evated to an exciting new level by the discoveries of hierarchical structures having at least two length scales, micro- and nanometer.$^{1-7}$ The ability to control structures in both length scales will have crucial impacts on biomineralization and design of nanomaterials. ${ }^{8-10}$ The micrometer level complexity of structure was first realized in chemical synthesis by Ozin et al. by using vesicles to sculpt inorganic shapes and patterns. ${ }^{11}$ Hollow microspheres of mesoporous silica have been obtained by condensing silica/surfactant assembly around a spherical organic phase, ${ }^{2,12}$ or micronsized spheres of mesoporous silica could be formed through aggregation. ${ }^{13}$ However, topological transformation producing various genus ranks ${ }^{14}$ has not been reported in a well-defined inorganic system. The ability to produce topological change in hierarchical matter is important toward understanding biomineralization. F or example, the elaborate organization of the silica shells of marine radiolaria shows high topol ogical genus ranks.

We report in this paper a preparation of mesoporous MCM-41 silica of hierarchical order, using surfactant, cosurfactant, and silicates. The use of a medium chain

* E-mail: cymou@ms.cc. ntu.edu.tw. Fax: 886-2-23636359.

(1) (a) Lin, H. P.; Mou, C. Y. Science 1996, 273, 765. (b) Lin, H. P.; Cheng, S.; Mou. C. Y. Chem. Mater. 1998, 10, 581. (c) Lin, H. P.; Cheng, S.; Mou, C. Y. Microporous Mater. 1997, 10, 111.

(2) Schacht, S.; Huo, Q.; Voigtmartin, I. G.; Stucky, G. D.; Schuth, F. Science 1996, 273, 768.

(3) (a) Tanev, P. T.; Pinnavaia, T.J. Science 1996, 271, 1267. (b) Tanev, P. T.; Liang, Y.; Pinnavaia, T. J . J . Am. Chem. Soc. 1997, 119, 8616.

(4) (a) Yang, H.; Coombs, N.; Ozin, G. A. Nature 1997, 386, 692. (b) Ozin, G. A.; Yang, H.; Sokolov, I.; Coombs, N. Adv. Mater. 1997, 9, 662.

(5) Davis, S. A.; Burkett, S. L.; Mendelson, N. H.; Mann, S. Nature 1997, 385, 420.

(6) Mann, S. Chem. Mater. 1997, 9, 2300.

(7) (a) Aksay, I. A.; Trau, M.; Mann, S.; Honma, I.; YaoZhou, N. L.; Fenter, P.; Eisenberger, P. M.; Gruner, S. M. Science 1996, 273, 892. (b) McGrath, K. M.; Dabbs, D. M.; Yao, N.; Aksay, I. A.; Gruner, S. M. Science 1997, 277, 552.

(8) Mann, S.; Ozin, G. A. Nature 1996, 382, 313.

(9) Ozin, G. A. Acc. Chem. Res. 1997, 30, 17.

(10) Trau, M.; Yao, N.; Kim, E.; Xia, Y.; Whiteside, G. M.; Aksay, I. A. Nature 1997, 390, 674.

(11) Oliver, S.; Kueperman, A.; Coombs, N.; Lough, A.; Ozin, G. A. Nature 1995, 378, 47.

(12) Singh, P. S.; Kosuge, K. Chem. Lett. 1998, 102

(13) Qi, L.; Ma, J.; Cheng, H.; Zhao, Z. Chem. Mater. 1998, 10, 1623.

(14) Topol ogical genus rank, $g$, is a measure of its connectedness of a closed surface. Two surfaces are of the same rank if one can be continuously deformed into another without any cutting. Hollow spheres (or tubules) belong to rank zero $(\mathrm{g}=0)$. For a doughnut, or equivalently a sphere with a handle, $\mathrm{g}=1$. For a pretzel (sphere with two handles) or its topological deformation, $\mathrm{g}=2$, and so on. For an introduction, please see: Hildebrandt, S.; Tromba, A. The Parsimonious Universe: Shape and Form in the Natural World; Springer-Verlag: New York, 1996.

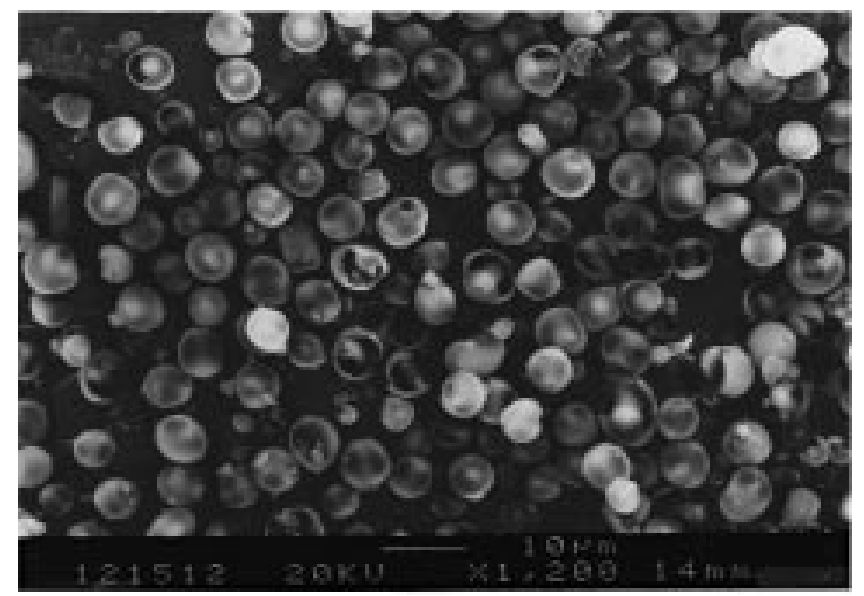

Figure 1. Scanning electron micrograph showing the morphology of the calcined sample. The sample is prepared from the gel mixture at $300 \mathrm{~K}$, after acidification, without any aging, followed by 2 days of hydrothermal treatment. Gel composition in molar ratio is 1:1.50:1.20:0.48:1.44:230 C14TMAB:SiO $\mathrm{NaOH}: \mathrm{H}_{2} \mathrm{SO}_{4}: \mathrm{BuOH}: \mathrm{H}_{2} \mathrm{O}$. Some broken spheres reveal that there is a pillar inside the spheres. The micrograph was taken on a J EOL J SM-6300 microscope with an accelerating voltage of $20 \mathrm{kV}$.

alcohol as cosurfactant is responsible for helping the bending elasticity of the liquid crystalline intermediate in micron-level structural transformations.

To prepare the sample, the delayed neutralization procedure previously reported ${ }^{1}$ is used. Here we use the cosurfactant butanol in addition to the surfactant myristyltrimethylammonium bromide (C14TMAB). The silica source is sodium silicate ${ }^{15}$ and the temperature is controlled at $300 \mathrm{~K}$. A suitable amount of butanol was added to the initial surfactant/sodium silicates solution to produce a two-phase solution. The upper phase is viscous and contains most of the surfactant, and the lower phase is a dilute micellar solution. Acid is slowly added to the well-stirred two-phase mixture to produce the surfactant-silica composite. The rest of the procedure is the same as reported before. ${ }^{1}$

Figure 1 is a SEM micrograph of the hollow spheres made after cal cination. The yield is rather high (>98\%) and the size is uniform. The average diameter is $5.0 \pm$ $1.0 \mu \mathrm{m}$. In Figure 1, some of the spheres as broken and they are al ways hollow with a pillar in the center. The spheres are intact after calcination; they are broken only when pressed hard by a spatula during SEM sample preparation. The X-ray diffraction (XRD) pattern of the material is typical for hexagonal MCM-41 with $\mathrm{d}_{100}=$ $3.7 \mathrm{~nm}$ (Figure 5E).

Figure $2 \mathrm{~A}$ shows an enlarged picture of one of the pillar-within-sphere (PWS) structures. Notice that the shell of the sphere appears rather thin, like an eggshell, and a fair amount of silica is located at the center pillar. Topologically, this new PWS structure (genus 1) is different from our previous tubules-within-tubule (TWT)

(15) The molar ratio of the resultant gel composition is (1.0) C14TMAB, (2.10-1.30) $\mathrm{SiO}_{2},(1.63-1.20) \mathrm{NaOH},(0.67-0.40) \mathrm{H}_{2} \mathrm{SO}_{4}$, $(0.6-2.0) \mathrm{BuOH}$, and (100-400) $\mathrm{H}_{2} \mathrm{O}$. After neutralization is complete, the gel undergoes a hydrothermal reaction at $100{ }^{\circ} \mathrm{C}$ for 2 days. 

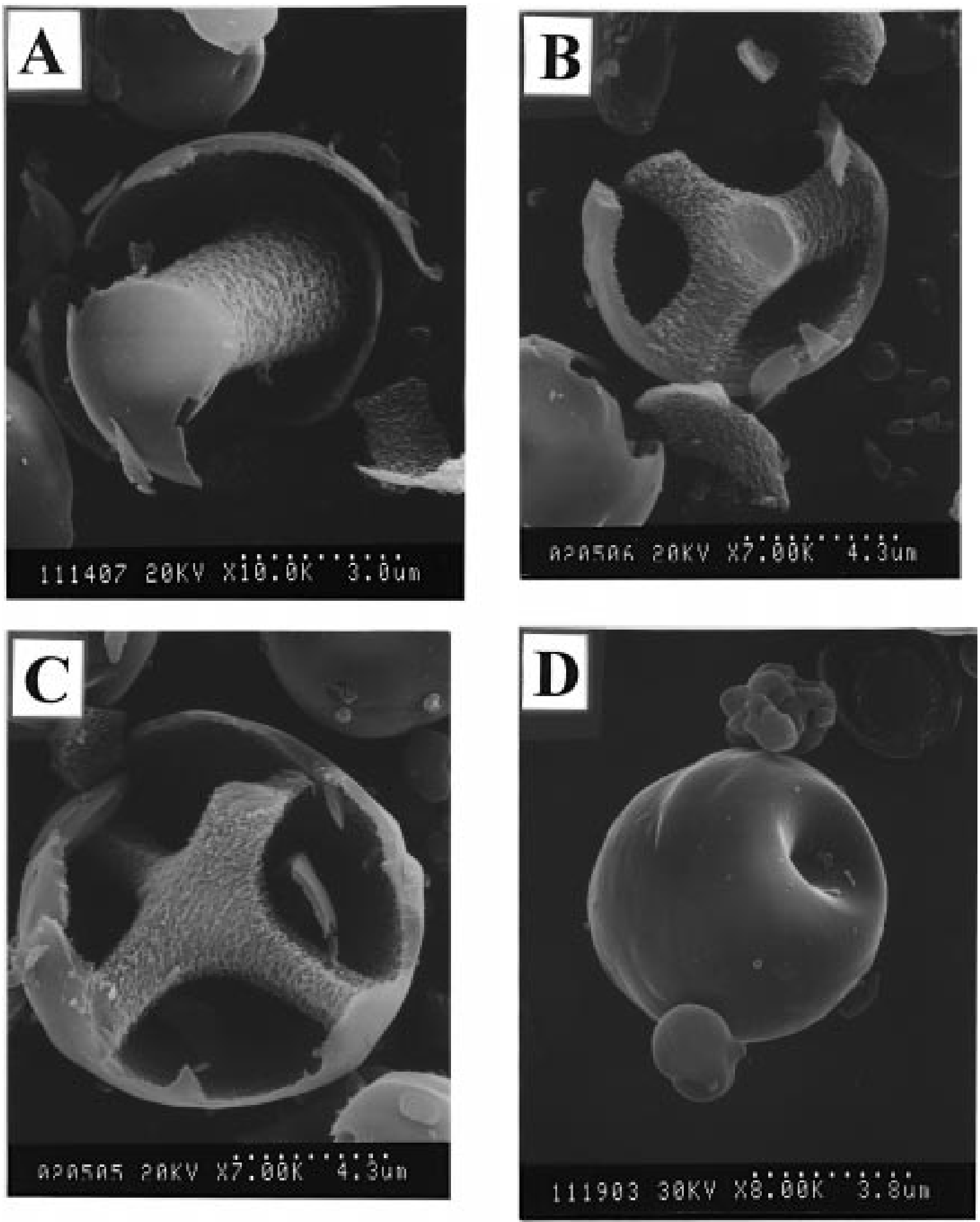

Figure 2. Scanning electron micrographs of spheres with different pillar shape. (A) pillar-within-spheres (genus = 1); (B) forkwithin-spheres (genus = 2); (C) cross-within-spheres (genus = 3); (D) An intact sphere with a dimple on the pole. These micrographs were taken on a Hitachi S-800 microscope with an accelerating voltage of $20 \mathrm{kV}$.

order. ${ }^{1}$ The TWT structure is a micron-sized hollow tubule whose wall consists of nanometer-sized coaxial channels of MCM-41 silica. Since it can be continuously distorted to a hollow sphere, the genus rank of TWT is zero. While most of the structures are of the PWS form, in some of the less frequent cases we find even higher genus structures. Figure $2 \mathrm{~B}$ is a picture for the forkwithin-sphere (FWS) structure with genus 2. The shell appears to be similar to that of the PWS, but the inside pillar becomes a fork supporting the spherical shell. Figure 2C shows a cross-within-sphere (CWS) structure with genus 3. The abundance of the three types is roughly in the order PWS $\gg F W S \cong C W S$. If the hydrothermal reaction is carried out at the elevated temperature of $150{ }^{\circ} \mathrm{C}$, the CWS becomes much more abundant. In Figure 2D, we show that the outside appearance of many of the spheres is not smooth. There are dimples on them that are the bases of pillar poles.

To gain a clear picture of the orientation of the hexagonal nanochannels, a transmission electron micrograph (TEM) of the calcined spherical pure silica MCM-41 materials is shown in Figure $3 \mathrm{~A}$. The picture 

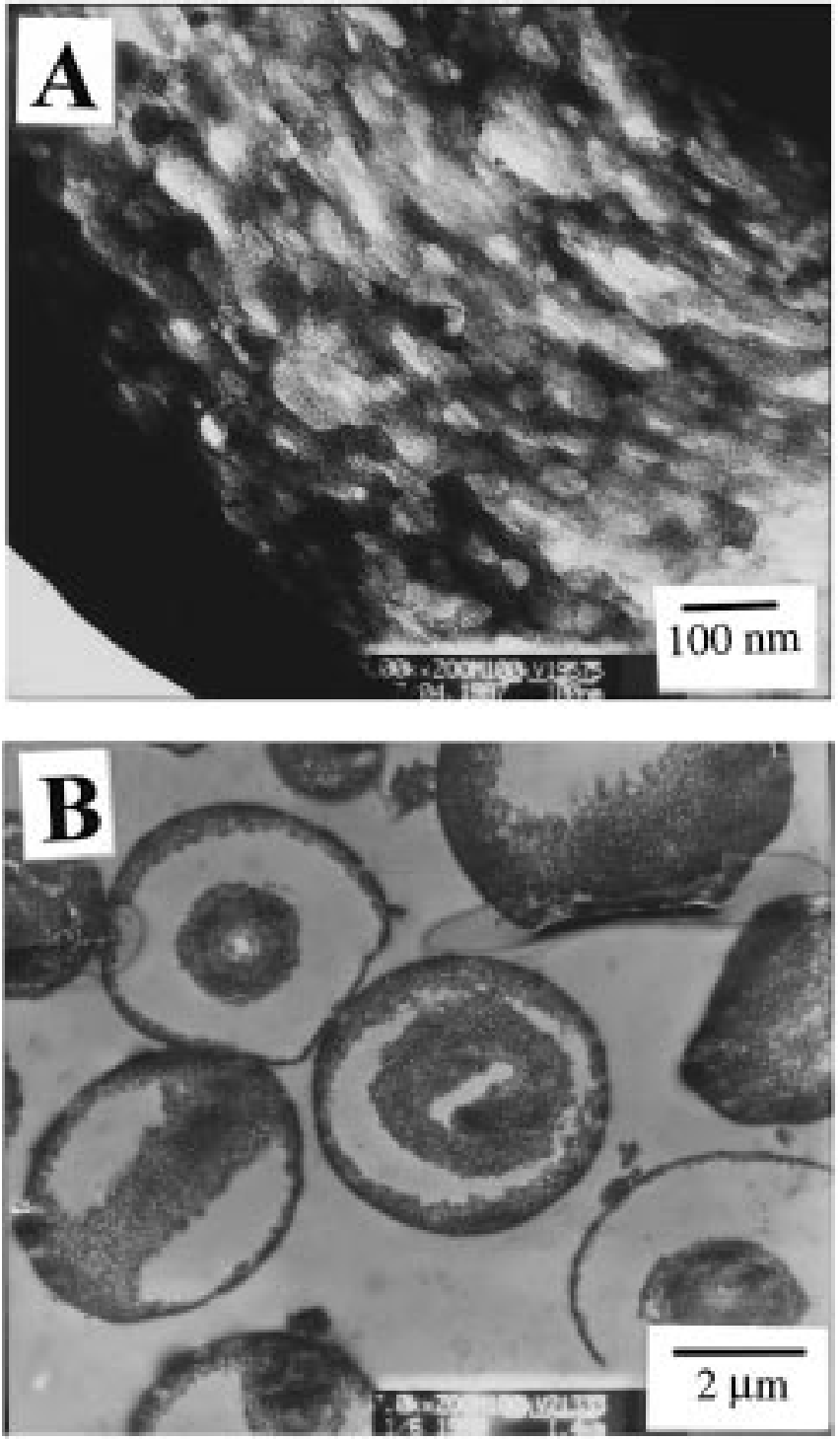

Figure 3. Transmissi on electron mi crograph (Hitachi $\mathrm{H}-7100$ TEM) of the calcined spherical pure silica MCM-41 material obtained under the same synthetic conditions as the sample shown in Figure 1. (A) Sample prepared by dispersing the powder material in water and then depositing and drying on a honeycomb carbon film on a Cu grid. (B) I mage obtained by embedding the sample in Spurr resin and then cutting into ultrathin sections.

is taken along the direction of the pillar; part of the center pillar is at the upper-right corner and the shell is in the opposite corner; in between one sees parallel nanochannels going al ong the latitude direction. The distance between the nanochannels agrees with that calculated from the XRD spectrum. From the SEM pictures in Figure 2, one can see that the channels also go around the outside wall of the pillars in the latitude direction. Figure 3B is a TEM picture of the ultrathin section of the PWS; at the center we see two spheres, and the cuts reveal that the pillars are indeed hollow. Topologically, the PWS is doughnut-like. Upon viewing the dimples in Figure 2D, one can see that the inside of the center pillar is partly hollow , but an excess amount of silicates would often fill up this region to make it solid. However, we sometimes do observe in TEM images a completely hollow pillar.
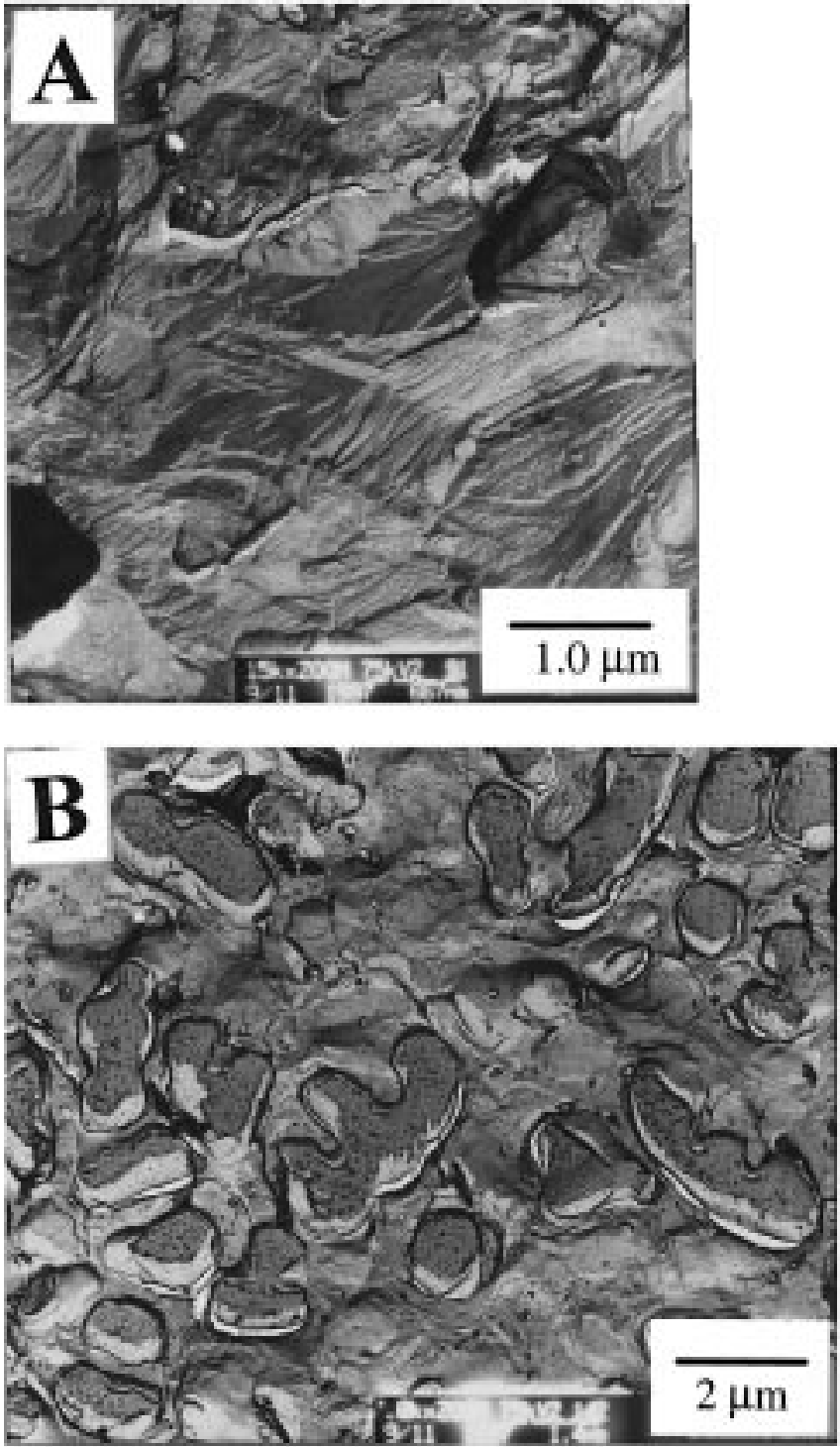

Figure 4. The freeze-fracture TEM (Hitachi H-7100 TEM) of the surfactant rich phase from the reaction intermediate [1:2.03:1.62:x:1.44:230 C14TAB: $\mathrm{SiO}_{2}: \mathrm{NaOH}: \mathrm{H}_{2} \mathrm{SO}_{4}: \mathrm{BuOH}: \mathrm{H}_{2} \mathrm{O}$, (A) $x=0,(B) x=0.10]$. (A) The unneutralized sample prepared at $300 \mathrm{~K}$ shows extensive layered membrane structures with a little interdispersed small vesicles. (B) After slight acidification at $300 \mathrm{~K}$, the sample shows more vesicles sitting in the layers.

Next, we want to look for the precursor of the spheres in the liquid crystal phase. We took freeze-fracture (FF) micrographs of the viscous gel phase of the initial two phases. Figure 4A, for the initial samples before any acidification, shows layered structures together with a little vesicles present in the gel. Because of the problem of unknown viewing angle and fracture action, it is not possible to estimate the vertical repeating distance of the layers from Figure 4A. It is certainly larger than the $2-3 \mathrm{~nm} d$ spacing for MCM-41. The layers are from a swollen lamellar phase. In recent years, there have been reports ${ }^{16,17}$ on swollen lamellar phases of surfactant systems. They are stabilized by an entropy-driven undulation motion of the membranes

(16) Larche, F. C.; Appell, A.; Porte, G.; Bassereau, P.; Marignan, J . Phys. Rev. Lett. 1986, 56, 1700.

(17) Schomacker, R.; Strey, R. J . Phys. Chem. 1994, 98, 3908. 
or electrostatic interactions. ${ }^{18} \mathrm{Alcohol}$ cosurfactants are known to soften the bilayers, e.g. the bending rigidity is reduced after the addition of alcohols. ${ }^{19-21}$ As a result, an enhanced undulation repulsion between bilayers may enable the swollen lamellar phase to change into tubular or spherical vesicle phases. Previously, we reported a TWT hierarchical order for MCM-41, and a "liquid crystal phase transformation" mechanism for the change from anisotropic membrane (consisting of a hexagonal phase) to tubule was proposed. (See Figure 4 of ref 1a.) By inserting cosurfactant alcohol molecules in the silicate-surfactant bilayers, we have delayed the hexagonal phase formation in the membrane, and thelayers become isotropic such that spherical vesicles instead of tubules are formed.

U pon slight acidification, the gel phase becomes very viscous and its FF-TEM micrographs (Figure 4 B) show that many vesicles embedded in the membranes are found. The shape is irregular, indicating low interfacial energy. However, we are not sure whether the vesicles are unilamellar or multilamellar. Previously, vesicles embedded in the lamellar phase has been observed for the dilute tertiary system CTAB (cetyltrimethylammonium bromide)/hexanol/brine ${ }^{22}$ or DMPC (dimyristoylphosphotidyl chloride)/geraniol/water. ${ }^{21}$ The embedding of vesicles in lamellar phase explains the high viscosity. In the beginning, the shape is nonspherical and the size of the vesicles is rather nonuniform, as shown in Figure 4B. But upon further acidification, they gradually change into uniform, micron-sized spherical vesicles. ${ }^{23}$ It seems that coagulation results in the uniform spherical vesicles with a fixed spontaneous curvature. One also notices in Figure $4 B$ that the inside of the vesicles looks grainy. By comparison with FFmicrographs of a silica sol solution, we conclude that the inside of the veside contains a high concentration of silica solution with nanoparticles of silica. When viewing by TEM, the inside of the vesicle appears to be dark, also indicating a high concentration of silica. So this implies that the early sequence of the process is swollen lamellar $\rightarrow$ embedded irregular vesicle $\rightarrow$ uniform spherical vesicle containing a silicate solution inside.

To understand the evolution of the nanostructures, we also analyzed the XRD of the intermediate liquid crystalline phases at various stages in the neutralization process. Figure $5 \mathrm{~A}$ is the XRD result of the gel mixture before any acidification. It gives a swollen lamellar phase, as revealed in the FF-micrograph in Figure 4A. The $d$ spacing $(4.1 \mathrm{~nm})$ is a bit bigger than the one without butanol $(2.6 \mathrm{~nm})$. It is due to the swelling of the lamellar phase. After $15 \%$ acidification, some silica condensation starts to occur and the $d$ spacing of the lamellar phase shrinks to $3.8 \mathrm{~nm}$. (Figure 5B) At 30$50 \%$ acidification, most of the lamellar structures disappear and the diffraction becomes very diffuse and weak

(18) Helfrich, W. Z. Naturforsch. 1978, 33a, 305.

(19) Safinya, C. R.; Sirota, E. B.; Roux, D.; Smith, G. S. Phys. Rev. Lett. 1989, 62, 1134.

(20) Ponsinet, V.; Fabre, P. J . Phys. Chem. 1996, 100, 5035.

(21) Chiruvolu, S.; Warriner, H. E.; Naranjo, E.; Idziak, S. H.; Rädler, J . O.; Plano, R. J .; Zasadzinski, J . A.; Safinya, C. R. Science 1994, 266, 1222.

(22) Gomati, R. J . Phys. Chem. 1987, 91, 6203.

(23) I mages from optical microscopy, not shown here, reveal uniform spheres with temperature-dependent diameter in the range of microns.

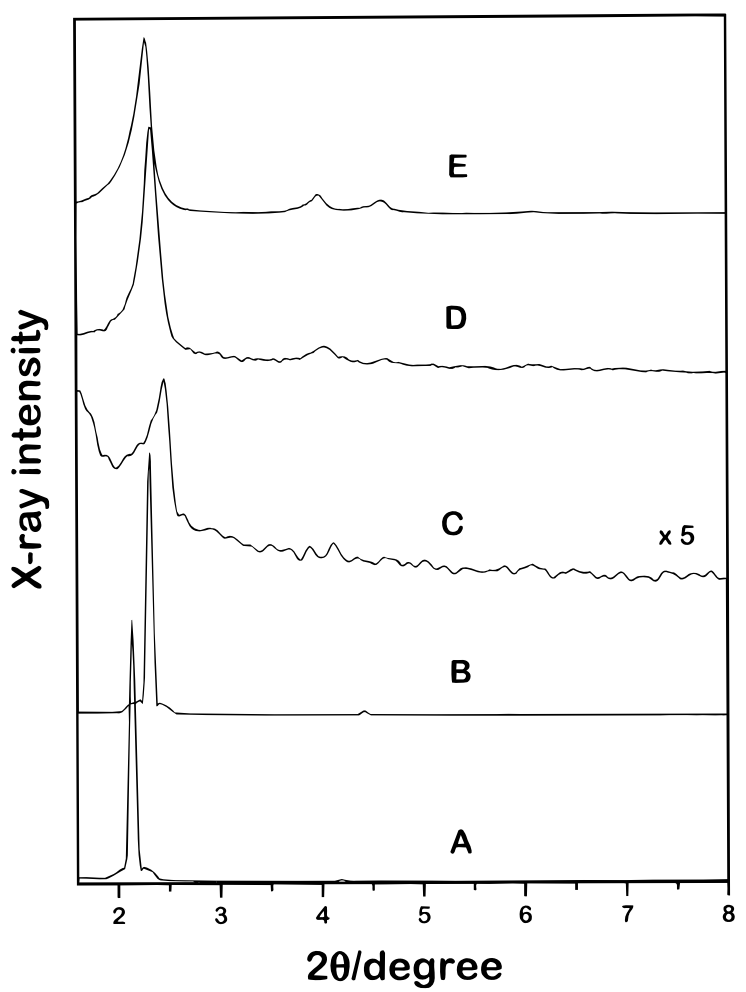

Figure 5. The XRD patterns of mesostructures synthesized from the same component at different stages of synthesis: 1:1.50:1.20:x:1.44:230 C14TMAB: $\mathrm{SiO}_{2}: \mathrm{NaOH}: \mathrm{H}_{2} \mathrm{SO}_{4}: \mathrm{BuOH}$ : $\mathrm{H}_{2} \mathrm{O},(\mathrm{A}) \mathrm{x}=0,(\mathrm{~B}) \mathrm{x}=0.07,(\mathrm{C}) \mathrm{x}=0.20$, (D) $\mathrm{x}=0.48$, and $(\mathrm{E})$ after $100^{\circ} \mathrm{C}$ hydrothermal reaction and cal cination of sample $D$. The patterns were col lected on a Scintag X1 diffractometer with $\mathrm{Cu} \mathrm{K} \alpha$ radiation $(\lambda=0.154 \mathrm{~nm})$.

(Figure $5 \mathrm{C}$ is magnified five times). TheFF-TEM image of Figure $4 \mathrm{~B}$ roughly corresponds to the stage when the lamellars transform into vesicles and finally disperse into the aqueous phase, thus becoming less viscous. This is because the lamellar phase holding the vesicle in the gel is gone. At $75 \%$ acidification, the transformation to the hexagonal phase is obvious but not quite complete (Figure 5D) and the cal cined sample of the final gel has al most the same hexagonal structure (Figure $5 E$ ) as the as-synthesized sample. Since initially we have vesicles, the final finite thickness of the shell of the spheres probably grows from the initial thin shell of the vesicles.

Previously, Michalet and Bensimon ${ }^{24}$ observed the equilibrium shapes of phospholipid vesicles in solution to be various topological genus, and they were able to understand them by accounting for the minimization of the elastic energy of a homogeneously curved surface. ${ }^{25}$ In our case, the shape transformation in pillar formation is assumed to occur as a consequence of a lamellar-to-hexagonal phase change on the shell of the spheres al ong latitude directions (Figure 6C). When the hexagonal channels grow to the pole region, the energy penalty of channels bending at the poles becomes very high. Although a curved surface morphology in MCM41 resulting from disinclination and dislocation is known, ${ }^{26}$ apparently the local curvature energy near the poles becomes too large (in fact singular) for the poles

(24) Michalet, X.; Bensimon, D. Science 1995, 269, 666.

(25) Porte, G. J.'Phys. Condensed Matter 1997, 4, 8649. 1887. 
Pillar-within-Spheres
(A)

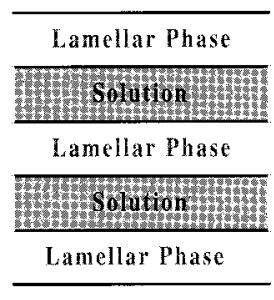

(D)

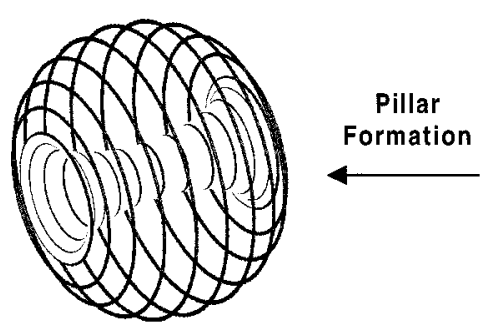

(B)

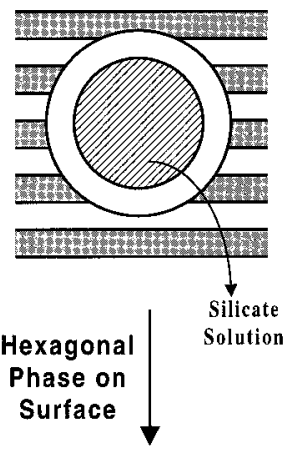

(C)

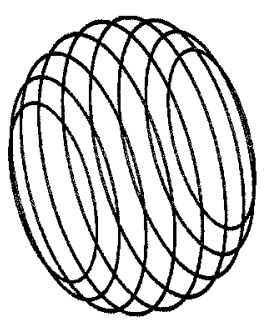

Figure 6. Proposed mechanism for the formation of the pillarwithin-spheres (PWS) morphology of MCM-41: (A) solvent swollen lamellar phase (the thickness of the layers are not known at the present) formed before acidification of the initial gel mixture. (B) More acidification induces the lamellar-tovesicle transformation and growth of shell thickness. At this stage, the shells are in lamellar states. (C) Partial hexagonal nanochannels formed on the shell of the spheres. (D) The hexagonal phase grows toward poles and could not close the channels at the poles. This leads finally to the formation of the center hollow pillar to relieve the energy singularity at poles.

to cl ose flatly as a hexagonal phase. Upon hydrothermal reaction, the excess silicates inside the sphere then condense to grow insi de the center hollow pillar in order to relieve the curvature energy at the poles. We only observe the pillar after the hydrothermal treatment. One then obtains a topological genus 1 pillar-withinspheres (PWS) structure, as shown in Figure 6D. Since, the nucleation of thelamellar-to-hexagonal change could be locally random and the windings of the nanochannels need not be completely around one single direction, it is not surprising that one could observe that the inside pillars are forked or crossed. There is a mi nor amount of these higher genus structures compared to the PWS structure at $100^{\circ} \mathrm{C}$, but their presence increases during higher temperature hydrothermal treatment. This is reasonable since one expects more nucleation of the hexagonal phase at higher temperature.

To summarize, mesoporous MCM-41 silica of hierarchical order was assembled from a solution of C14TMAB, butanol, and sodium silicate by careful control of the acidification rate. The products consist of a high yield ( $98 \%$ ) of uniform-sized hollow spheres of diameter 5.0 $\pm 1.0 \mathrm{~m}$. The inside of the micron-sized spheres has an intricate pillar structure possessing various topological genus ranks. The walls of these microspheres consist of a hexagonal arrangement of nanochannels in latitude directions. The whole process is driven by the following physicochemical transformation: silica condensation driving a membrane-to-vesicle and then a lamellar-to-hexagonal transformation on the shell, in turn driving pillar formation. The yield is high and the morphology is uniform since it is based on an equilibrium phase change. Our findings, in view of the recent developments in theories of complex fluids, ${ }^{25}$ suggest that the preparation of hierarchical order materials by the sol-gel method can be approached as a physicochemical phase transformation process. When the underlying physical chemistry is better understood, the method can be rationally designed. This new development is important not only for understanding biomineralization but also for discovering new hierarchical matter for applications. Since our PWS structures are very uniform in size, they could be the building blocks for forming two-dimensional arrays of colloidal particles for potential applications as photonic devices. ${ }^{27}$

Acknowledgment. Electron microscopy work by Mr. C. Y. Tang is gratefully acknowledged. We are thankful for discussions with Prof. S. B. Liu, B.-Z. Wan, D. Lu, S. Cheng, and Dr. F. Y. Lee. This research was supported by the China Petroleum Co. and the National Science Council of Taiwan (NSC 85-2113-31-M-002-032 CC).

\section{CM980493V}

(27) Kim, E.; Xia, Y.; Whitesides, G. M. Adv. Mater. 1996, 8, 245. 